\title{
Erratum
}

\section{Foliation Theory in Algebraic Geometry}

\author{
Paolo Cascini, James $\mathrm{M}^{\mathrm{c}}$ Kernan, and Jorge Vitório Pereira
}

Mathematics Department, Imperial College of London, London, UK

Department of Mathematics, UC San Diego, La Jolla, CA, USA

IMPA, Rio de Janeiro, Brazil

(c) Springer International Publishing Switzerland 2016

P. Cascini et al. (eds.), Foliation Theory in Algebraic Geometry, Simons Symposia,

DOI 10.1007/978-3-319-24460-0

\section{DOI 10.1007/978-3-319-24460-0_8}

The original version of the book contained errors which have been corrected in an updated version.

The corrections are given below:

\section{Chapter 1}

In the original version of Chapter 1 , in page 4 , fourth paragraph should be read as below:

Given a sheaf $\mathscr{F}$ of $\mathscr{O}_{X}$-modules on a variety $X$, we denote by $\mathscr{F}^{*}$ the sheaf $\mathscr{H}_{0} m_{\mathscr{O}_{X}}\left(\mathscr{F}, \mathscr{O}_{X}\right)$. If $r$ is the generic rank of $\mathscr{F}$, then we $\operatorname{denote}$ by $\operatorname{det}(\mathscr{F})$ the sheaf $\left(\wedge^{r} \mathscr{F}\right)^{* *}$. For $m \in \mathbb{N}$, we denote by $\mathscr{F}[m]$ the sheaf $\left(\mathscr{F}{ }^{\otimes m}\right)^{* *}$. If $\mathscr{G}$ is another sheaf of $\mathscr{O}_{X}$-modules on $X$, then we denote by $\mathscr{F}[\otimes] \mathscr{G}$ the sheaf $(\mathscr{F} \otimes \mathscr{G})^{* *}$.

On page 12, under section 3.3, "Hör 13" in second paragraph should be read as "Hör 14". Also, "Hör 13" in the reference list is now changed as "Hör 14".

The online version of the original chapter can be found under http://dx.doi.org/10.1007/978-3-319-24460-0_1

\section{Chapter 2}

The second affiliation of F. Bogomolov is now updated as "National Research University Higher School of Economics, Russian Federation, AG Laboratory, HSE, 7 Vavilova str., Moscow 117312, Russia” 
On page 37, line 6, "I.3.4" should be read as "1.3.3".

On page 46, line 5, "II.1.1" should be read as "2.1.1".

The online version of the original chapter can be found under http://dx.doi.org/10.1007/978-3-319-24460-0_2

\section{Chapter 5}

On page 108 , line 16 , " $\mathcal{C}_{x}(X)$ " has been changed as " $\tau_{x}: \mathcal{U}_{x} \rightarrow \mathcal{C}_{x}(X)$ ".

On page 123, last seven lines have been replaced as:

In the other long root cases the VMRTs of the model manifold are linearly degenerate, and we made use of the more general result that $\mathcal{W}$ must be bracket generating for a uniruled projective manifold $X$ of Picard number 1 to reach the same conclusion in [HM02]. Here we say that a distribution $\mathcal{W}$ on $X$ is bracket generating to mean that the tangent subsheaf generated by $\mathcal{W}$ from taking successive Lie brackets is the tangent sheaf. The same resoning was applied to the short-root cases in [HM04a] and [HM05]. In the cases of [HM05], the VMRTs of the model spaces are linearly nondegenerate.

On page 134, section 4.4.1, "i" in the second line has been italicized.

On page 135, "i" in the first line has been italicized.

On page 138, in fourth paragraph, third from the last line " $\mathcal{C S}$ " has been changed as "C $(\mathcal{S})$ ".

On page 138, Definition 4.5.4 has been updated with the below text:

Definition 4.5.4. Let $\varpi: \mathcal{C}(S) \rightarrow S, \mathcal{C}(S):=\mathcal{C}(X) \cap \mathbb{P} T(S)$, be a subVMRT structure on $S \subset X-B^{\prime}$ as in Definition 4.5.1. For a point $x \in S$, and $[\alpha] \in \operatorname{Reg}\left(\mathcal{C}_{x}(S)\right) \cap \operatorname{Reg}\left(\mathcal{C}_{x}(X)\right)$, we say that $\left(\mathcal{C}_{x}(S),[\alpha]\right)$, or equivalently $\left(\widetilde{\mathcal{C}_{x}}(S), \alpha\right)$, satisfies Condition $(\mathrm{T})$ if and only if $T_{\alpha}\left(\widetilde{\mathcal{C}_{x}}(S)\right)=T_{\alpha}\left(\widetilde{\mathcal{C}_{x}}(X)\right) \cap T_{x}(S)$. We say that $\varpi: \mathcal{C}(S) \rightarrow S$ satisfies Condition $(\mathrm{T})$ at $x$ if and only if $\left(\widetilde{\mathcal{C}}_{x}(S),[\alpha]\right)$ satisfies Condition (T) for a general point $[\alpha]$ of each irreducible component of $\operatorname{Reg}\left(\mathcal{C}_{x}(S)\right) \cap \operatorname{Reg}\left(\mathcal{C}_{x}(X)\right)$. We say that $\varpi: \mathcal{C}(S) \rightarrow S$ satisfies Condition (T) if and only if it satisfies the condition at a general point $x \in S$.

The online version of the original chapter can be found under http://dx.doi.org/10.1007/978-3-319-24460-0_5 